\title{
Documenting the need for routine influenza vaccination in kenya; a case of influenza associated severe acute respiratory illness outbreak, nakuru county, kenya
}

\begin{abstract}
Severe Acute respiratory illness continue to be the leading cause of acute illnesses worldwide and remain the most important cause of morbidity in children less than one year. Influenza A and B viruses are among the most common causes of respiratory tract illnesses that bring children to medical care. Influenza surveillance in Kenya describes the incidence and prevalence of influenza among patients with ILI, SARI, and ARI. Influenza accounts for up to a quarter of ILI cases, a tenth of SARI cases and up to half of all ARI cases. Influenza vaccination is the main strategy of prevention, but influenza vaccination is not in the Routine immunization Schedule in Kenya. The purpose of this study therefore is to demonstrate the burden of influenza associated SARI and document the need for introduction of influenza vaccine into the routine immunization schedule. The study was conducted in Nakuru county referral hospital located in Nakuru County which is one of the 47 counties of the Republic of Kenya. The county lies within the Great Rift Valley. A retrospective analysis of SARI outbreak data that was collected during the outbreak between March and June 2016. Case definition was used to identify cases. SARI cases were entered into an excel line list. Data Analysis was done using SPSS version 20. Chi square tests were used to establish association of relevant risk factors with the occurrence of influenza associated SARI. All confidence intervals were maintained at 95\%. Statistical significance was defined at $p \leq 0.05$. P value of $\leq 0.05$ was considered significant. The outbreak began in week 9 of 2016 in Nakuru North as epi centre and eventually spread to the entire County. A total of 823 cases with 94 deaths were reported. All sexes were equally affected. Children less than 2 years were mostly affected and high mortality recorded amongst children less than one year. Nasopharyngeal swabs Samples were analysed in 5 laboratories. Results indicated that $42 \%$ were as a result of RSV, $30 \%$ influenza A and B, $12 \%$ did not yield any pathogen and the rest were as a result of other viruses. Important risk factors identified included Co-morbidities $\left(\mathrm{X}^{2}\right.$ 1716.7, $\mathrm{p}$ 0.000), non vaccination with influenza vaccine and malnutrition $\left(X^{2} 1379, \mathrm{p} 0.000\right)$. The prevalence of Influenza among sari patients is high, comorbidities, non vaccination and malnutrition are the major risk factors. It is necessary to introduce influenza vaccine into routine immunization schedule and to carryout another study to determine the prevalence of malnutrition and associated factors so as to form the basis for intervention.
\end{abstract}

Keywords: severe acute respiratory illness, influenza, risk factors, influenza vaccine, co-morbidities
Volume 3 Issue I - 2016

\author{
Elizabeth Kiptoo,' Florence Basweti, ${ }^{2}$ Jane \\ Kinoti, ${ }^{3}$ Samuel King ori' \\ 'Department of Health Services, Kenya \\ ${ }^{2}$ Nakuru County Referral Hospital, Kenya \\ ${ }^{3}$ Njoro Sub County Health Office, Kenya
}

Correspondence: Elizabeth Kiptoo, Department of Health Services, Nakuru, Kenya, Tel 254722337018 ,

Email lizkiptoo@gmail.com

Received: October 10,2016 | Published: October 31, 2016
Abbreviations: ARI, acute respiratory infection; CEC, county executive committee; $\mathrm{CDC}$, centers for diseases control; $\mathrm{CHO}$, chief officer health; CSF, celebral spinal fluid; DALYs, daily adjusted life years; ILI, influenza like illness; KEMRI, kenya medical research institute; NPHLS, national public health laboratories; RSV, respiratory syncitial virus; SARI, severe acute respiratory illness; SPSS, statistical package for social science; WRP, walter reed program

\section{Introduction}

Severe Acute respiratory infection (ARIs) continue to be the major cause of acute illnesses globally and remain the most important cause of sickness in children less than one year and young. ${ }^{1}$ They account for up to two million deaths a year and rank first among causes of disability adjusted life years (DALYs) lost in developing countries (94.6million) $6.3 \%$ of total. ${ }^{2}$ The incidence of SARIs in children less than five years is estimated to be 0.29 and 0.05 episodes per child per year in third world and developed countries respectively which translates to 151 million and 5 million new cases each year. ${ }^{3}$ Influenza surveillance in Kenya describes the incidence and prevalence of influenza among patients with ILI, SARI, and ARI. Influenza accounts for up to a quarter of ILI cases, a tenth of SARI cases and up to half of all ARI cases. ${ }^{4}$

In Kenya, it is estimated that influenza resulted in 57,000 to 81,000 cases of severe respiratory illnesses in 2009 and between 960 to 1,420 deaths over the course of the year. ${ }^{5}$ The population most at risk for developing a fatal respiratory disease are the very young, the elderly and the immune-compromised. ${ }^{3}$ Influenza A and $\mathrm{B}$ viruses are among the most common causes of respiratory tract illnesses that bring children to medical care, and influenza is a major cause of lower respiratory tract illness in young children. ${ }^{6}$ The annual incidence of influenza infection in children may exceed 30 percent and children are believed to be important in the spread of influenza in the community. ${ }^{7}$ In march to June 2016 an outbreak of Influenza associated SARI was reported in Nakuru County Referral hospital. A 
total of 823 cases with 94 deaths were reported. Influenza vaccination is the main strategy of prevention, but influenza vaccination is not in the Routine immunization Schedule for children less than one year. The purpose of this study therefore is to demonstrate the burden of influenza associated SARI and document the need for introduction of influenza vaccine into the schedule of Routine Immunization in order to prevent mortality and morbidity associated with Influenza.

Vaccination is the primary influenza prevention strategy. ${ }^{8}$ The vaccine is inactivated and is prepared yearly with strains chosen on the basis of information provided by a worldwide surveillance network about the strains that are believed likely to circulate widely during the upcoming season. ${ }^{9,10}$ Its efficacy is 70 to 90 percent in preventing disease. ${ }^{9}$

\section{Methods}

\section{Study site}

The study was conducted in Nakuru county referral hospital located in Nakuru County which is one of the 47 counties of the Republic of Kenya. The county lies within the Great Rift Valley and borders seven other counties namely; Kericho to the west, Baringo and Laikipia to the north, Nyandarua to the east, Narok to the southwest and Kajiado and Kiambu to the south. The county covers an area of 7,495.1Km.It is divided into eleven administrative Sub-Counties namely; Naivasha, Gilgil, Nakuru East, Nakuru west,Rongai, Nakuru North, Subukia, Njoro, Molo, Kuresoi North and Kuresoi South. The total population of Nakuru County stands at 2,087,950 in 2016. There are 354 health facilities and immunization coverage of children 12 23 months is $85 \%$ (KDHS 2009).

\section{Study design}

A retrospective analysis of SARI outbreak data that was collected during the outbreak between March and June 2016.

\section{Case definition}

A suspected case of SARI is any case less than five years with an acute respiratory infection with history of fever or measured fever of $\geq 38^{\circ} \mathrm{C}$ and cough with onset within the last 10days and requires hospitalization.

\section{Data collection and analysis}

SARI cases were entered into an excel line list. The line list captured socio demographic variables that included the, name, age, sex, date of onset, date seen at the health facility/hospital, residence, vaccination status, nutrition status, laboratory results and outcome of the illness. Data Analysis was done using SPSS version 20. Chi square tests for categorical variables were used to establish association of relevant risk factors with the occurrence of influenza associated SARI. Reported p-values were two-sided and all confidence intervals (CI) were maintained at $95 \%$. Statistical significance was defined at $\mathrm{p}$ $\leq 0.05$. $\mathrm{P}$ value of $\leq 0.05$ was considered significant.

\section{Ethical approval}

The study was part of routine disease surveillance and outbreak investigation by the Department of Health services Nakuru county and ethical approval was not considered necessary because this was a retrospective analysis of data that already existed.

\section{Results}

All the 11 Sub counties in Nakuru County were affected The out break initially began in Nakuru East, Nakuru West and Nakuru North and spread to the other 9 sub counties eventually affecting the entire County (Figure 1). The neighbouring countries of Baringo, Nyandarua and Narok were not spared either. In total 823 cases were reported with 94 deaths (CFR 11.4\%).The records indicated that the outbreak picked in week 9 of 2016 (Figure 2). Laboratory results Implicated the following viruses; influenza virus type A and B, RSV and Parainfluenza. All sexes were equally affected. It mostly affected children less than 2years and high mortality recorded amongst children less than one year (Figure 3).

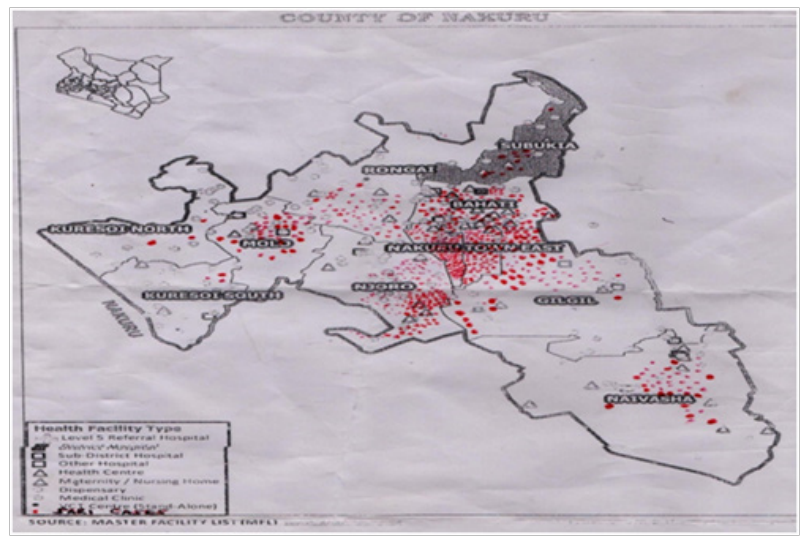

Figure I Distribution of Sari cases.

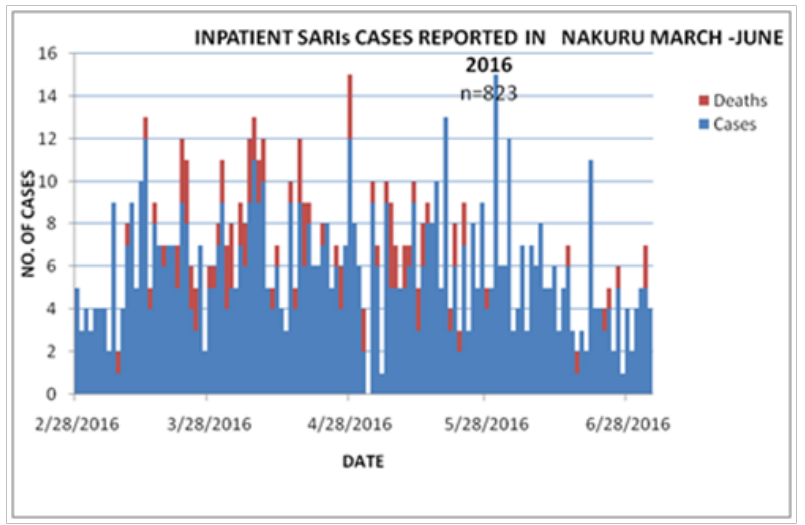

Figure 2 Epi curve of SARI outbreak, Nakuru county, Kenya 2016.

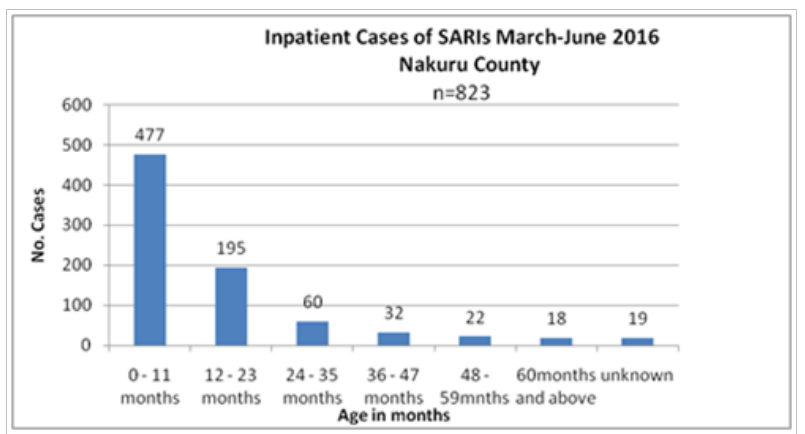

Figure 3 Distribution of SARI cases by age.

\section{Virological lab results}

Samples were collected from 74 patients and analysed in 5 laboratories KEMRI/WRP Flu lab, CDC lab (Nairobi), CDC lab (Kisumu), WRP lab (Kisumu) and the NPHLS labs (Nairobi) for quality control. The rest of the cases were epidemiologically linked to the confirmed cases. Genetic sequencing done on two samples positive for RSV subtype B revealed an identity to RSV viruses that 
were circulating in 2013 and 2014 in Guangzhou, China influenza type A virus revealed identity to Influenza A viruses in Czech Republic 2016(H1N1) and India 2015(H1N1) (Figure 4).

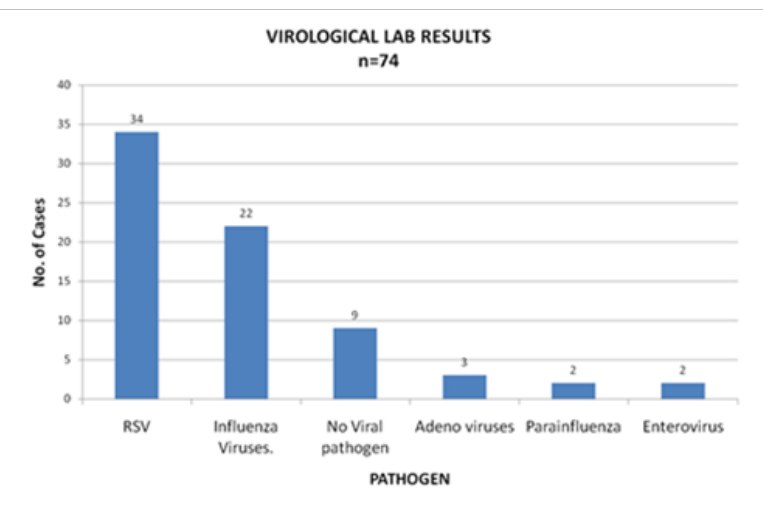

Figure 4 Virological laboratory results.

\section{Bacteriology lab results}

Samples collected included blood, cerebral spinal fluid (CSF), and stool, did not yield pathogenic bacterial isolates due to prior patient treatment with antibiotics. Post mortem samples - tracheal swab, lung swab, and aspirates did not detect viruses on them but yielded Klebsiella pneumoniaeandEscherichia coli which were resistant to first and second line cephalosporins and penicillins and sensitive to carbapenems (Imepenem and Meropenem).

\section{Histopathology - autopsy}

Clinical autopsy performed on 5 cases in Nakuru county referral hospital: Gross examination was done by pathologists and scientists in KEMRI/CDC influenza Division. Specimens included lung, bronchi, Heart, spleen, liver, kidneys, brain and skeletal muscles. Two (2) cases showed features characteristics of co infection by viral and bacterial etiology. One case showed necrotizing pneumonia which is characteristics of staphylococcal pneumonia. ${ }^{11}$ Last case was limited by lack of representative specimens of the lung, however necrotic splenic micro abcesses and cocci are consistent with staphylococcus aurius strain and is frequently preceded by influenza infection. One was found that the patient had RSV and influenza B viruses.

\section{Discussion}

This outbreak is the first of its kind to occur in the country. It occurred between the months of march through june 2016. Nakuru East, Nakuru West and Nakuru North where the outbreak began is densely populated and are neighbouring one another then quickly spread to the other nine sub counties (Figure 3). The study has two major findings. First, Laboratory confirmed influenza was associated to $30 \%$ of total cases of Severe Acute Respiratory Illness (SARI). This proportion is significant and cannot be ignored and is higher than the results of a similar study carried out in Nepal in which laboratoryconfirmed influenza was associated with nearly $10 \%$ of overall respiratory hospitalizations. ${ }^{6}$ The findings are similar with the findings of Clinical and virological surveillance data of acute respiratory infections among children less than 5years old living in rural areas in Senegal which indicated that influenza represented about $30 \%$ of positive viral detection in patients with ARI. ${ }^{12}$ These cases and deaths could be averted by vaccinating the children with the flu vaccine. Children less than five years were affected with majority in the age bracket of children less than two years and mortality mainly occurring in children less than on year. All the affected had not had any influenza vaccination. This finding is similar to the findings of a study carried out in El Salvador that found out those children aged $<5$ years in El Salvador are frequently hospitalized as a result of influenza. ${ }^{5}$ The findings still is in agree with the findings of another study carried out in Erbil governorate of Iraq that found out that younger children were more likely to have ARI with children less than one year being mostly affected. ${ }^{13}$ However, this finding is different from the findings of a Global Pooled Analysis of risk Factors for H1N1pandemic, The highest per capita risk of hospitalization was among patients above 5 and between 5-14years old respectively, whereas the highest risk of death per capita was in the 50-64 and $>65$ year old age groups. ${ }^{14}$ Secondly other important risk factors identified included Comorbidités including HIV infection, chronic Lung and heart diseases and malnutrition. Household characteristics (overcrowding, indoor cooking, parental smoking) were not significant this is contrary to the findings of a A case-control study carried out in Ibadan Nigeria among houses of children under the age of 5 years that associated overcrowding, parental smoking and indoor cooking with Acute respiratory infections. ${ }^{15}$

Chi square Pearson revealed a strong association between the occurrence of influenza associated SARI and malnutrition $\left(\mathrm{X}^{2} 1379\right.$, p 0.000), Comobidity ( $\left.X^{2} 1716.7, p 0.000\right)$ and of course non vaccination with flu vaccine $\left(\mathrm{X}^{2} 1702, \mathrm{p} 0.000\right)$. Other studies have also associated ARI with the said risk factors. Chalabi in his case control study found out that children who are underweight were more likely to have SARI than the control group. ${ }^{13}$ In addition Tupasi in his study found out that malnourishment was associated with increased risk of SARI Morbidity. ${ }^{16}$ An annual seasonal flu vaccine is the best way to reduce the chances that one gets seasonal flu and spread it to others. When more people get vaccinated against the flu, less flu can spread through that community. ${ }^{2,17}$

\section{Conclusion}

The prevalence of Influenza among SARI patients is high, comobidities and malnutrition are the major risk factors. It is necessary to introduce influenza vaccine into routine immunization schedule and to carryout another study to determine the prevalence of malnutrition and associated factors so as to form the basis for intervention.

\section{Acknowledgements}

This study was supported by Ministry of Health National Government and the department of health services Nakuru County Government. We thank Our colleagues from the said institutions Dr. Philip Muthoka, Dr. Emmanuel Okunga and Rosalia Kalani who provided insights and expertise that greatly assisted in the study.

We thank Professor Wallace Bulimo of KEMRI/USAMRD-K and his team, Laboratory experts from KEMRI/WRP Flu lab, CDC lab (Nairobi), CDC lab (Kisumu), WRP lab (Kisumu) and the NPHLS labs (Nairobi) for Their expertise assistance in laboratory investigation.

We would also like to show our gratitude to Dr. K. Mungai CEC; Health Services, Dr. S. Mwaura; COH Dr. S. Sirma Director Medical services, Dr. J. Lenai Director Public Health, Dr. B. Osore Director Administration and planning and Nakuru County Heath Management Team for their technical and financial support. We are also immensely greatful to all the Sub county Health management teams, Hospital Management teams and all health workers Nakuru County for their support in one way or another. 


\section{Authors' contributions}

All Authors participated in outbreak investigation, as well as drafting and approval of manuscript. EK analysed the data.

\section{Recommendation for another study}

This study recommends another study to be done on prevalence of under nutrition and associated factors.

\section{Conflicts of interest}

Author declares there are no conflicts of interest.

\section{Funding}

None.

\section{References}

1. Gyawal M, Pahari R, Maharjan S, et al. Knowledge on acute respiratory infection among Mothers of under five year children of Bhaktapur District, Nepal. International Journal of Scientific and Research Publications. 2016;6(2):2250-3153.

2. NCPD NC. Kenya Population situation analysis. Nairobi: Government of Kenya, Africa. 2013.

3. Nissen MD, Lambert SB, Whiley DM, et al. Respiratory infections. Brisbane: Springer science, USA. 2010. p.67-68.

4. Katz MA, Muthoka P, Emukule GO, et al. Results From the First Six Years of National Sentine 1Surveillance for Influenza in Kenya, July 2007-June 2013. PLoS One. 2014;9(6):e98615.

5. Clara W, Armero J, Rodriguez D, et al. Estimated incidence of influenzavirus-associated severe pneumonia in children in El Salvador, 20082010. Bulletin of World Health Organization. 2012. p.756-763.

6. Belshe RB, Mendelman PM, Treanor J, et al. The efficacy of Attenuated, cold adapted, Trivalent, Intranasal influenza virus in children. $N$ Engl $J$ Med. 1998;338(20):1404-1412.
7. Ji W, Zhang $\mathrm{T}$, Zhang $\mathrm{X}$, et al. The epidemiology of hospitalized influenza in children, a two year population-based study in the People Republic of China. BMC Health Serv Res. 2010;10:82.

8. Ferdinands JM, Olsho LE, Agan AA, et al. Effectiveness of Influenza Vaccine Against Life-threatening RT-PCR-confirmed Influenza Illness in US Children, 2010-2012. Journalof infectious Diseases. 2014;210(5):500-508.

9. Barnett, Elizabeth D. Influenza Vaccination for Children. The New England Journal of Medicine. 1998;338(20):1461.

10. WHO. Observed Rate of vaccine reactions influenza vaccine. World Health Organization. 2012. p.1-8.

11. Löffler B, Niemann S, Ehrhardt C, et al. Pathogenesis of Staphylococcus aureus necrotizing pneumonia: the role of PVL and an influenza coinfection. Expert Rev Anti Infect Ther . 2014;11(10):1041-1051.

12. Niang MN, Diop OM, Sarr FD, et al. Viral etiology of respiratory infections in children under 5 years old living in tropical rural areas of Senegal: The EVIRA project. J Med Virol. 2010;82(5):866-872.

13. Chalabi DK. Acute Repiratory infection and malnutrition among children below five years of age in Erbil governorate Irag. East Mediterr Health J. 2013;9(1):1-5.

14. Mounts WA, Kerkhove MD, Donnelly C. Risk Factors for H1N1pdm Severity. PMEDICINE. 2009;10:00373.

15. Ana GR, Morakinyo MO, Fakunle GA. Indoor Air Quality and Risk Factors Associated with Respiratory Conditions in Nigeria. Ibadan: INTECH. 2015.

16. Tupasi TE, Mangubat NV, Sunico ME, et al. Malnutrition and Acute Respiratory Tract Infections in Filipino Children. Journal of infectious diseases. 1990;8(12):1537-6591.

17. CDC. The single best way to protect against the flu is to get vaccinated each year. Key Facts About Seasonal Flu Vaccine. 2016. p.1-9. 